\title{
Iatrogenic Subtotal Stenosis of the Right Subclavian Artery Treated With Percutaneous Transluminal Angioplasty
}

\author{
Robert M. Smeenk • Mark C. J. M. Kock • \\ Otto E. H. Elgersma $\cdot$ Marco J. Schnater
}

Received: 9 July 2010/ Accepted: 13 August 2010/Published online: 16 September 2010

(C) The Author(s) 2010. This article is published with open access at Springerlink.com

\begin{abstract}
This report describes a rare vascular complication of surgical placement of a marking clip and a possible approach to problem solving. A 55-year-old patient presented with loss of sensation in the fingers and loss of peripheral pulsations in the right arm 4 days after right upper lobectomy for a pT2N1 moderately differentiated adenocarcinoma of the lung. Duplex examination and computed tomography were performed the same day and showed a subtotal stenosis of the right subclavian artery, which was caused by the surgical placement of a metal clip to mark the surgical boundary. Selective angiography was subsequently performed. Percutaneous transluminal angioplasty (PTA) successfully dilated the stenosis and pushed the clip off. Flow in the right subclavian artery (RSA) was completely restored as were neurology and peripheral pulses. In conclusion, arterial stenosis by a surgical (marking) clip may be feasibly treated with PTA.
\end{abstract}

Keywords Arterial intervention - Angioplasty/

Angiogram $\cdot$ Endovascular treatment $\cdot$ Artery

\section{Introduction}

Surgical clips are widely used in modern surgery. In addition to vascular and endoscopic clips, surgeons use metal clips liberally for marking purposes. Surgical

\section{R. M. Smeenk $(\bowtie) \cdot$ M. J. Schnater}

Department of Surgery, Albert Schweitzer Hospital, Albert Schweizterplaats 25, 3318 AT Dordrecht, The Netherlands e-mail: r.m.smeenk@asz.nl

M. C. J. M. Kock · O. E. H. Elgersma

Department of Radiology, Albert Schweitzer Hospital, Albert Schweizterplaats 25, 3318 AT Dordrecht, The Netherlands boundaries are marked for future radiotherapy in various oncologic surgical procedures. To our knowledge, complications caused by placement of surgical marking clips have not been described before. This case report illustrates a complication of the placement of a surgical marking clip in the thoracic cavity and subsequent successful treatment.

\section{Case Report}

A 55-year-old man presented with cold fingers and numbness of the right hand 4 days after undergoing right upper lobectomy for a pT2N1 moderately differentiated adenocarcinoma of the lung. The patient's history did not show any vascular pathology.

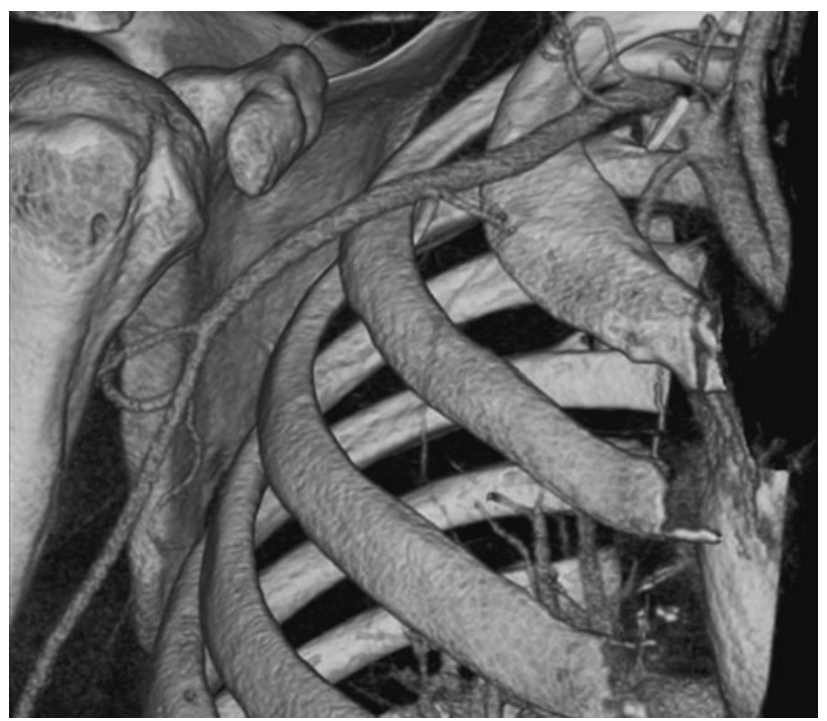

Fig. 1 Multiplanar reformat reconstruction of a CT image showing subtotal stenosis of the RSA caused by a surgical marking clip 
Physical examination showed a significant temperature difference between the fingers of the right and the left hand. Radial, ulnar, and brachial artery pulses were absent, and a slight loss of sensation was noted. Motor functions were intact.

On the same day, duplex examination was performed and showed a $>50 \%$ stenosis of the RSA. The peak systolic velocity proximal and distal to the stenosis was $142 \mathrm{~cm} / \mathrm{s}$ and $756 \mathrm{~cm} / \mathrm{s}$, respectively. There was no visible cause of stenosis. Subsequently, computed tomography angiography (CTA) of the upper thorax and right arm was performed. This showed subtotal stenosis of the RSA adjacent to a metal clip placed just distally to the origin of the vertebral artery (Fig. 1). This surgical clip was placed to mark the upper boundary in the thoracic cavity, where the tumor was adhesive to the parietal pleura, for possible future radiotherapy.

The patient was immediately admitted to the angiography suite to perform selective angiogram of the RSA through a groin approach (Fig. 2). Subtotal stenosis of the RSA was confirmed. The stenosis was passed using a glidewire (Terumo Europe, Leuven, Belgium) and a 5F Headhunter catheter (Boston Scientific, Nieuwegein, the Netherlands). Subsequently, the glidewire was replaced for an Amplatz guidewire (Boston Scientific, Nieuwegein, the Netherlands).
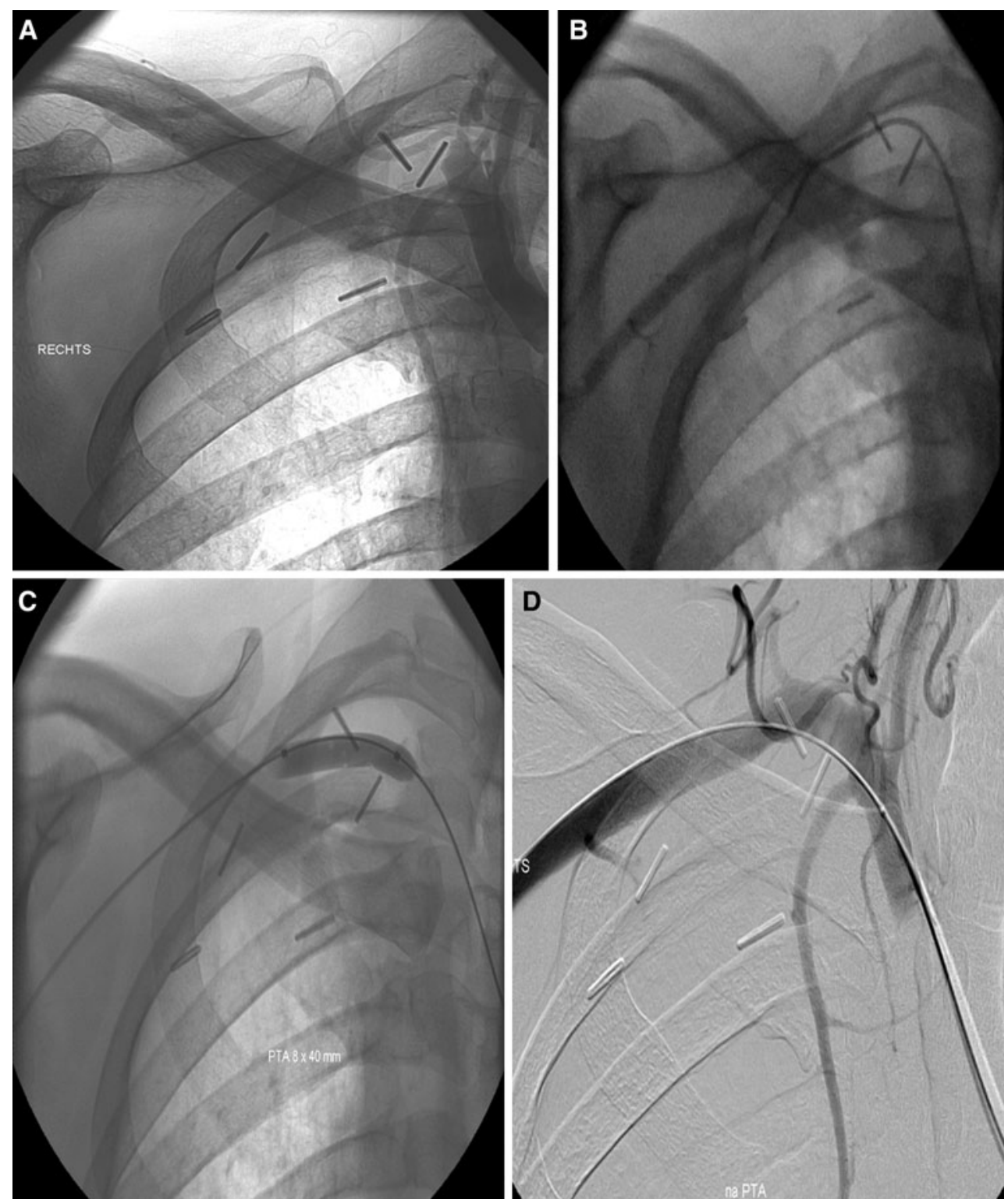

Fig. 2 A Selective angiogram of the RSA showing a metal clip on the artery just distally to the origin of the vertebral artery. B Angiogram of the RSA showing passage of a catheter through the stenosis with intraluminal contrast injection to confirm its position

distally to the stenosis. C Angiogram of the RSA showing the 8-mm balloon insufflated. D Angiogram of the RSA showing restoration of blood flow and displacement of the surgical clip 
A stiff catheter with a subtle tip was used to pass the curve of the aortic arch. The catheter was replaced for a semicompliant 8-mm PTA balloon catheter (Optapro; Cordis). The balloon diameter was chosen after measurement of the normal lumen of the RSA distal to the stenosis on CT. The stenosis was dilated to $8.5 \mathrm{~mm}$, determined by an insufflation table, thereby pushing the surgical clip off the artery caudally. This was seen on fluoroscopy. Leaving the guidewire in place, the balloon was removed and replaced by a $6 \mathrm{~F}$ monorail catheter (Heart Medical Europe B.V, Best, the Netherlands), which was placed with its tip at the origin of the RSA. Selective contrast injections showed an undamaged and patent RSA. A covered stent (Fluency Vascular Graft, Bard Benelux N.V., Nieuwegein, the Netherlands) was available in case of dissection, but the intervention was successful and uncomplicated, and the catheter and guidewire were subsequently removed. Neurology and peripheral pulses recovered. The responsible thoracic surgeon was informed. At follow-up in the outpatient ward, the patient was free of the previous symptoms in his right arm.

\section{Discussion}

Arterial stenosis caused by placement of a surgical clip is an uncommon complication, with only one report in literature [1]; however, it did not involve a marking clip as in this case report. Usually, surgical marking clips are placed under direct vision. Fortunately, surgical marking clips are not applied as tightly as vascular clips, the purpose of which is to occlude blood vessels. This might have been the reason for the late onset of symptoms and mild clinical presentation in this case. Thus, it was assumed that the misplaced clip might be pushed off the RSA from within instead of performing a second thoracotomy to extract the clip. Of course, the backup plan would be a second thoracotomy to extrude the clip or, in the case of a complication by the endovascular procedure, to save the RSA.

The only case report on this topic describes a surgically obstructed left internal mammary artery graft by a clip after coronary bypass surgery. PTA successfully treated this complication. There are some more reports on vascular stenosis by medical devices, but these describe complications after vascular closing devices, such as the Starclose Closure device [2-4]. In these cases, the complication was treated surgically.
In the presented case, a surgical marking clip, placed in the upper boundary of the surgical resection of the upper pulmonary lobe, caused subtotal stenosis of the RSA. During the surgical procedure, the misplacement of the clip was not recognized because it was placed far up in the thoracic cavity. Thus, the origin of stenosis was thought to be atherosclerotic, and CTA was performed first instead of performing angiography directly. Of course, when thinking of an obstruction caused by corpora aliena, one could even have first performed CT with dual-energy imaging. CTA then unexpectedly showed the surgical marking clip on the RSA. Subsequently, a selective angiogram was performed, and the clip was successfully pushed away with use of an angioplasty balloon.

After this incident in our institution, we have discussed the use of surgical marking clips in oncologic upper thoracic surgery, but for now there are no other means for other marking techniques in our institution.

\section{Conclusion}

Arterial stenosis caused by a surgical marking clip may feasibly be treated first with an endovascular procedure, consisting of an angiogram with PTA, before performing a second surgery.

Conflict of interest The authors declare that they have no conflict of interest.

Open Access This article is distributed under the terms of the Creative Commons Attribution Noncommercial License which permits any noncommercial use, distribution, and reproduction in any medium, provided the original author(s) and source are credited.

\section{References}

1. Klein AL, Marquis JF, Higginson LA (1988) Percutaneous transluminal angioplasty of a surgically obstructed left internal mammary artery graft. Catheter Cardiovasc Diagn 14:46-48

2. Bent CL, Kyriakides C, Matson M (2008) Femoral artery stenosis following percutaneous closure using a StarClose closure device. Cardiovasc Interv Radiol 31:814-816

3. Eidt JF, Habibipour S, Saucedo JF et al (1999) Surgical complications from hemostatic puncture closure devices. Am J Surg 178:511-516

4. Stone PA, Campbell JE, Andrews KH et al (2008) Posterior wall capture and resultant common femoral occlusion complicating StarClose access closure. J Vasc Surg 48:469-471 